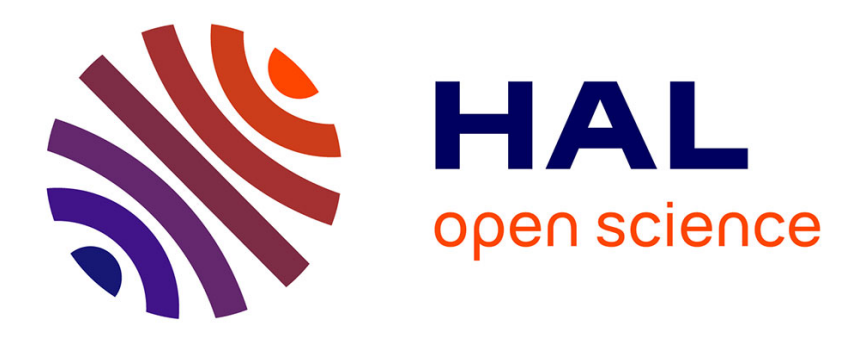

\title{
Note sur la capacité d'ingestion des vaches laitières en fin de lactation
}

\author{
J.P. Dulphy, J. Rouel
}

\section{To cite this version:}

J.P. Dulphy, J. Rouel. Note sur la capacité d'ingestion des vaches laitières en fin de lactation. Productions Animales, 1988, 1 (2), pp.93-96. hal-00895820

\section{HAL Id: hal-00895820 \\ https://hal.science/hal-00895820}

Submitted on 1 Jan 1988

HAL is a multi-disciplinary open access archive for the deposit and dissemination of scientific research documents, whether they are published or not. The documents may come from teaching and research institutions in France or abroad, or from public or private research centers.
L'archive ouverte pluridisciplinaire HAL, est destinée au dépôt et à la diffusion de documents scientifiques de niveau recherche, publiés ou non, émanant des établissements d'enseignement et de recherche français ou étrangers, des laboratoires publics ou privés. 
INRA Prod. Anim., 1988, 1 (2), 93-96

\author{
J.P. DULPHY \\ J. ROUEL \\ INRA Theix \\ Station de Recherches \\ sur la Nutrition des Herbivores \\ 63122 Ceyrat \\ * INRA Domaine d'Orcival \\ 63210 Rochefort-Montagne
}

\section{Note sur la capacité d'ingestion des vaches laitieres en fin de lactation.}

La capacité d'ingestion des vaches laitières varie de façon progressive tout au long de leur cycle gestationlactation. La bibliographie sur ce point est relativement claire : citons par exemple Journet et Remond (1976) et Forbes (1977). La capacité d'ingestion des vaches est minimale juste après le vêlage, augmente jusque vers la $12^{e}$ semaine après (Faverdin et al 1987) puis diminue très lentement jusqu'au tarissement. Cependant, en fin de lactation, cette capacité d'ingestion n'est pas bien connue car, à cette époque, les animaux sont en général au pâturage. C'est pourquoi, durant 2 années, nous avons mesuré les quantités ingérées par des vaches en fin de lactation. Ces mesures ont été faites après une conduite normale au pâturage, ce qui n'est jamais présenté dans la littérature, pour un vêlage en février-mars. Elles concernent des ensilages d'herbe alors que les résultats bibliographiques concernent en général des foins ou des ensilages de maïs.

Dans ces essais des animaux en début de lactation. jusqu'à la $12^{\mathrm{e}}$ semaine après le vêlage, ont été pris comme témoins. Ces animaux étaient différents des animaux en fin de lactation, mais il est très difficile de comparer un même régime pendant $\mathbf{1 0}$ mois. Ce protocole a donc permis d'avoir en même temps des vaches en

\section{Résumé}

Les quantités ingérées par des vaches laitières en fin de lactation ont été mesurées pour une ration à base d'ensilage d'herbe et comparées à celles mesurées en début de lactation (respectivement semaines 34-46 après le vêlage et semaines 1 à 12). Au maximum d'ingestion ( $5^{\mathrm{e}}$ semaine après le vêlage) les vaches produisant $33 \mathrm{~kg}$ de lait à $4 \%$ de $M G$ ont ingéré $20,6 \mathrm{~kg}$ de MS (ensilage d'herbe + concentré) ; en fin de lactation, pour une production de $11 \mathrm{~kg}$ de lait ( $40^{\circ}$ semaine après le vêlage), elles ont ingéré $14,1 \mathrm{~kg}$ de $\mathrm{MS}$. Cette dernière valeur correspond à une capacité d'ingestion de 15,6 UEL, cohérente avec le système des unités d'encombrement. Il existe cependant des variations individuelles de la capacité d'ingestion en fin de lactation qui ne s'expliquent pas par le format ni par la production des animaux. début et en fin de lactation. En effet la capacité d'ingestion des vaches au $3^{e}$ mois de lactation est bien connue et devait nous permettre d'estimer la valeur d'encombrement du fourrage utilisé.

\section{Conditions expérimentales}

Chaque année, durant 2 ans, 2 lots de vaches, l'un en début, l'autre en fin de lactation ont reçu, ensemble, un même ensilage de dactyle. Les vaches en fin de lactation étaient rentrées à l'étable en septembre, les mesures étant effectuées jusqu'à fin décembre, époque du tarissement des animaux. Les vaches en début de lactation vêlaient en novembre et étaient suivies jusque fin janvier. Les principales conditions expérimentales sont résumées dans le tableau 1 et les caractéristiques des ensilages dans le tableau 2. Ces caractéristiques ne sont pas très différentes, le second ensilage étant cependant légèrement mieux conservé que le premier. Le premier a été distribué en outre à des moutons pour mesurer sa valeur alimentaire (ingestibilité $=55 \mathrm{~g}$ de $\mathrm{MS} / \mathrm{kg}$ $\mathrm{P}^{0.75}$; valeur énergétique $=0,85 \mathrm{UFL} / \mathrm{kg}$ de $\mathrm{MS}$; valeur azotée $=103 \mathrm{~g}$ de PDIN et $78 \mathrm{~g}$ de PDIE $/ \mathrm{kg}$ de MS). Les animaux étaient en stabulation entravée sans paille. Les quantités d'ensilage offertes et refusées ont été pesées 4 jours par semaine ( $10 \%$ de refus environ) et la production de lait a été mesurée chaque jour. Le lait a été analysé 2 jours par semaines. Enfin les animaux ont été pesés tous les mois, ainsi qu'en début et fin d'essai (double pesée).

\section{Résultats}

Les principaux résultats figurent, pour les périodesclés (début de lactation - semaines 1 à 8 , milieu de lactation - semaines 9 à 12 et fin de lactation - semaines 35 à 46) dans le tableau 3 pour chaque année et en moyenne par période dans la figure 1. Malgré des diffé- 
Tableau 1. Principales conditions expérimentales.

\begin{tabular}{|c|c|c|}
\hline $\begin{array}{l}\text { Essai } \\
\text { Année }\end{array}$ & $\stackrel{\text { I }}{1985-1986}$ & $\underset{1986-1987}{\text { II }}$ \\
\hline Ration & \multicolumn{2}{|c|}{$\begin{array}{l}\text { Ensilage de dactyle } 1^{\text {er }} \text { cycle à volonté }+ \text { aliment concentré et C.M.V. } \\
\text { pour couvrir les besoins. }\end{array}$} \\
\hline Concentré & \multicolumn{2}{|c|}{$\begin{array}{l}\text { Aliment composé de céréales }+ \text { pulpe de betterave }+ \text { tourteau, équilibré pour } \\
\text { la production de lait }(1,05 \mathrm{UFL} / \mathrm{kg} \text { de MS et } 122 \mathrm{~g} \text { de PDI })+\text { tourteau tanné } \\
\text { (soja }+ \text { colza). }\end{array}$} \\
\hline & \multirow{2}{*}{\multicolumn{2}{|c|}{$\begin{array}{l}\text { En début de lactation, augmentation progressive de } 1 \mathrm{~kg} \text { tous les } 3 \text { jours } \\
\text { jusqu'à un maximum fonction du potentiel des vaches, puis décroissance régu- } \\
\text { lière, en faisant l'hypothèse d'une diminution de la production de lait de } 2 \% \\
\text { par semaine et d'une couverture de } 10 \mathrm{~kg} \text { de lait par l'ensilage. } \\
\text { En fin de lactation : } \\
\text { au-dessus de } 12 \mathrm{~kg} \text { de lait }\end{array}$}} \\
\hline & & \\
\hline Animaux & \multicolumn{2}{|c|}{$\begin{array}{l}2 \text { lots différents de } 8 \text { vaches } \\
\text { multipares Ho et croisées Ho } \times \text { FF idem }\end{array}$} \\
\hline $\begin{array}{l}\text { Production de lait maximum } \\
\text { (kg brut/jour) }\end{array}$ & $\begin{array}{l}39 \text { (lot début) } \\
33 \text { (lot fin) }\end{array}$ & $\begin{array}{l}30 \text { (lot début) } \\
36 \text { (lot fin) }\end{array}$ \\
\hline $\begin{array}{l}\text { Stade de lactation } \\
\text { (en semaines) }\end{array}$ & $\begin{array}{l}0-14 \text { (lot début) } \\
34-45 \text { (lot fin) }\end{array}$ & $\begin{array}{l}0-12 \text { (lot début) } \\
35-46 \text { (lot fin) }\end{array}$ \\
\hline
\end{tabular}

Tableau 2. Caractéristiques des ensilages utilisés (ensilage direct, coupe fine + conservateur acide).

\begin{tabular}{|l|c|c|}
\hline Essai & I & II \\
Année & 1985-1986 & 1986-1987 \\
\hline Teneur en MS (\%) & 21.6 & 23.1 \\
Teneur en g/kg de MS & & \\
- matières azotées totales & 173 & 178 \\
- matières minérales & 105 & 97 \\
- cellulose brute & 300 & 273 \\
\hline pH & 4.65 & 4.30 \\
N-NH \% N total & 11.7 & 9.7 \\
N sol. \% N total & 57.5 & 59.4 \\
Acide lactique & 27 & 64 \\
Acide acétique $\}$ en g/kg & 64 & 36 \\
Acide butyrique de MS & 4 & 3 \\
Alcools & 25 & 11 \\
\hline
\end{tabular}

rences entre années le potentiel des animaux a été le même pour ceux en début et ceux en fin de lactation ( $34,5 \mathrm{~kg}$ de lait brut, soit $33 \mathrm{~kg}$ de lait à $4 \%$ de $M G$ ). La perte de poids en début de lactation a été relativement faible $(14 \mathrm{~kg}$ de poids vif en 1 mois et, au total, $6 \mathrm{~kg}$ en 3 mois, soit en poids vif corrigé respectivement 26 et $22 \mathrm{~kg}$ ). En fin de lactation le poids vif corrigé a augmenté de $25 \mathrm{~kg}$.

Les quantités d'ensilage ingérées sont passées de 11,6 $\mathrm{kg}$ de MS en $12^{\mathrm{e}}$ semaine à $12,7 \mathrm{~kg}$ en $34^{\mathrm{e}}$ semaine (pour des quantités de concentré égales à 8,6 et $1,5 \mathrm{~kg}$ ). Elles se sont ensuite maintenues autour de $13 \mathrm{~kg}$ pour chuter lors des 3 semaines avant le tarissement. Les quantités ingérées totales sont passées par un maximum de $20,6 \mathrm{~kg} \mathrm{de}$ MS au cours de la $5^{\mathrm{e}}$ semaine après le vêlage.

Pour un ensilage de dactyle au $1^{\text {er }}$ cycle (digestibilité mesurée de la $M O=72 \%$ ) les tables (INRA 1988) don- nent, par interpolation, une valeur d'encombrement de 1,11 pour un ensilage parfaitement conservé et 1,20 pour un ensilage sans conservateur. Compte tenu des teneurs élevées en acide acétique des ensilages utilisés nous retiendrons 1,15 - valeur très proche de celle estimée en 1986 à partir des quantités ingérées lors des semaines 9 à $12(1,17)$ selon les propositions de Faverdin et al (1987) pour la capacité d'ingestion des vaches. En 1985 par contre l'appétit des animaux n'était pas à son maximum et il n'est pas possible de vérifier la valeur proposée. On se basera donc simplement sur le fait que les caractéristiques des ensilages étaient peu différentes entre les 2 années.

Dans ces conditions, et en regroupant les effectifs des 2 années on trouve une capacité moyenne d'ingestion de 15,6 UEL en fin de lactation, contre 15,45, valeur proposée par Faverdin et al (1987) pour une production de lait à $4 \%$ de $M G$ égale à $11 \mathrm{~kg}$. 


\section{Discussion - Conclusion}

Bien qu'ayant pris des animaux différents en début et en fin de lactation nous obtenons des résultats cohérents à la fois avec ceux de la bibliographie et ceux de Faverdin et al (1987). Les vaches en fin de lactation que nous avons observées ont bien une capacité d'ingestion élevée qui leur permet d'être largement suralimentées $(+2,3$ UFL dans les 2 essais) et de reprendre du poids. Cette capacité d'ingestion est très stable, sauf lors des 3 dernières semaines avant le tarissement, alors que le vêlage approche. Par contre, les résultats obtenus en comparant les 2 années ne sont pas satisfaisants, car très différents, peut-être à cause d'un état d'engraisse. ment différent des animaux d'une année à l'autre (Bines et Morant 1983), facteur connu de modification des quantités ingérées en dehors du poids vif et de la production laitière.

En début de lactation les vaches ont ingéré les mêmes quantités, malgré des potentiels très différents. Par contre, il semble bien que les vaches fortes productrices aient mis plus de temps à atteindre leur capacité d'ingestion maximale, ce qui n'est pas surprenant (Jarrige 1986) puisque en semaines 9 à 12 les vaches de 1985 étaient encore en bilan énergétique négatif alors que celles de 1986 étaient en bilan largement positif. Dans ces conditions, la capacité d'ingestion réelle est plus faible que celle prévue à partir de la production laitière et la valeur d'encombrement déduite par le fourrage est surévaluée (on trouverait 1,38!).

Notons enfin que la baisse des quantités totales ingérées a été en moyenne de $800 \mathrm{~g}$ de MS par mois pendant 8 mois. Faverdin et al (1987) donnent une valeur de $1000 \mathrm{~g} /$ mois pour des rations à base d'ensilage de maiis, les valeurs de la bibliographie étant en moyenne de $500-700 \mathrm{~g} / \mathrm{mois}$ et pouvant descendre à $100 \mathrm{~g}$ pour certaines rations (Mohrenweiser et Donker 1968, Murdock et Hodgson 1969, Monteiro 1972, Brown et al 1977, Donker et Mac Clure 1982, Holter et al 1982, Donker et al 1983, Bywater 1984, Oldenbroek 1984).
Figure 1. Evolution des quantités ingérées, de la production laitière et du poids vif, en moyenne dans les 2 essais.

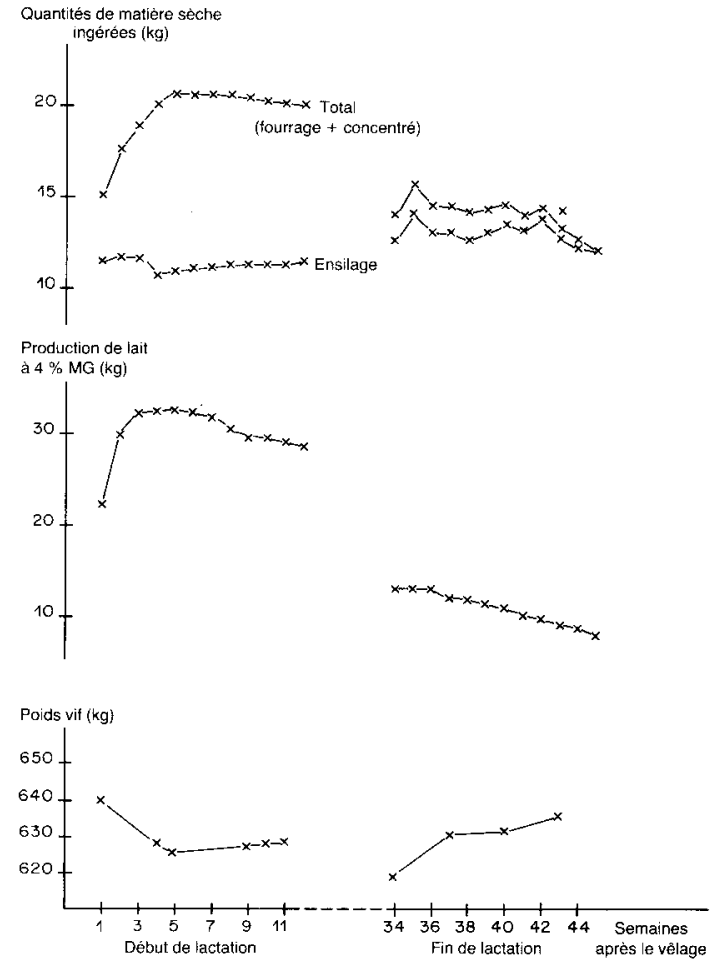

Tableau 3. Ingestion et production des vaches laitières

\begin{tabular}{|c|c|c|c|c|c|c|}
\hline $\begin{array}{l}\text { Essai } \\
\text { Année }\end{array}$ & & $\stackrel{I}{1}$ & & & $\underset{986-19}{\text { II }}$ & \\
\hline Semaines & $1-8$ & $9-12$ & $34-45$ & $1-8$ & $9-12$ & $35-46$ \\
\hline $\begin{array}{l}\text { Quantités de MS ingérées } \\
\text { - ensilage } \\
\text { - tourteau tanné } \\
\text { - concentré équilibré } \\
\text { TOTAL }\end{array}$ & $\begin{array}{r}11.12 \\
1.24 \\
7.20 \\
19.56\end{array}$ & $\begin{array}{r}11.49 \\
1.05 \\
8.18 \\
20.72\end{array}$ & $\begin{array}{r}14.00 \\
0.00 \\
0.78 \\
14.77\end{array}$ & $\begin{array}{r}11.48 \\
1.65 \\
6.16 \\
19.29\end{array}$ & $\begin{array}{r}11.34 \\
1.36 \\
7.05 \\
19.75\end{array}$ & $\begin{array}{r}12.27 \\
0.30 \\
0.90 \\
13.47\end{array}$ \\
\hline $\begin{array}{l}\text { Production de lait par jour } \\
\text { - quantité de lait (kg) : } \\
\text { - brut } \\
-4 \% \text { MG } \\
\text { - matières grasses (g) } \\
\text { - matières protéiques (g) }\end{array}$ & $\begin{array}{l}35.6 \\
34.4 \\
1326 \\
1042\end{array}$ & $\begin{array}{l}35.6 \\
33.0 \\
1234 \\
1004\end{array}$ & $\begin{array}{l}12.8 \\
12.1 \\
468 \\
389\end{array}$ & $\begin{array}{l}27.7 \\
26.9 \\
1057 \\
854\end{array}$ & $\begin{array}{l}28.3 \\
25.7 \\
958 \\
844\end{array}$ & $\begin{array}{c}9.6 \\
9.8 \\
398 \\
324\end{array}$ \\
\hline $\begin{array}{l}\text { Poids vif moyen }(\mathrm{kg}) \\
\text { Variation du poids vif }(\mathrm{kg}) \text { : } \\
\begin{aligned} \text { - non corrigé } \\
\text { - corrigé }\end{aligned}\end{array}$ & $\begin{array}{c}630 \\
-7 \\
-28\end{array}$ & $\begin{array}{l}634 \\
+6 \\
+4\end{array}$ & $\begin{array}{r}630 \\
+24 \\
+33\end{array}$ & $\begin{array}{l}635 \\
-20 \\
-29\end{array}$ & $\begin{array}{r}625 \\
+8 \\
+8\end{array}$ & $\begin{array}{r}633 \\
+14 \\
+16\end{array}$ \\
\hline
\end{tabular}


En définitive, nos essais permettent de préciser, dans le cadre d'une conduite classique et pour des ensilages de qualité moyenne, comment évolue l'appétit des vaches laitières en fin de lactation. Globalement les résultats sont compatibles avec les calculs de capacité d'ingestion et de valeur d'encombrement proposés dans le système des UE (Dulphy et al 1987). Cependant des différences sont toujours possibles, telles que l'appétit non encore maximal de vaches en bilan énergétique négatif et des variabilités individuelles de la capacité d'ingestion en fin de lactation ne s'expliquant pas seulement par le format et la production des animaux.

\section{Références bibliographiques}

BINES I.A., MORANT S.V., 1983. The effect of body conditions on metabolic changes with intake of food by cows. Brit. J. Nutr., $50,81.89$.

BROWN C.A., CHANDLER P.T., HOLTER J.B., 1977. Development of predictive equations for milk yield and dry matter intake in lactating cows. J. Dairy Sci., 60, 1739-1754

BYWATER A.C., 1984. A generalised Model of feed intake and digestion in lactating cows. Agricultural Systems, 13, 1189-1204.

DONKER J.D., Mac CLURE F.A., 1982. Responses of milking cows to amounts of concentrate in rations. J. Dairy Sci., 65, 1189-1204.

DONKER J.D., MARX G.D., YOUNG C.W., 1983. Feed intake and milk production from three rates of concentrate for cows bred to differ in size. J. Dairy Sci., 66, 1337-1348.
DULPHY J.P., FAVERDIN Ph., MICOL D., BOCQUIER F., 1987. Révision du système des unités d'encombrement. Bull. Tech. CRZV Theix, INRA, 70, 35-48.

FAVERDIN Ph., HODEN A., COULON J.B., 1987. Recommandations alimentaires pour les vaches laitières. Bull. Tech. CRZV Theix, INRA, 70, 133-152.

FORBES J.M. 1977. Development of a model of voluntary food intake and energy balance in lactating cows. Anim. Prod., 24 203-214.

HOLTER J.B., HYLTON W.E., SMITH C.B., URBAN Jr W.E., 1982. Reducing concentrate feeding for lactating dairy cows. J. Dairy Sci., 65, 37-51.

INRA, 1988. Alimentation des Bovins, Ovins, Caprins. R. Jarrige éd., 491 pages. Edit. INRA Publications, Versailles.

JARRIGE R., 1986. Voluntary intake in dairy cows and its prediction. International Dairy Federation. Bull. $\mathrm{n}^{\circ} 196,4-16$.

JOURNET M., REMOND B., 1976. Physiological factors affecting the voluntary intake of feed by cows : a review. Livestock Prod Sci., 3, 129-146.

MOHRENWEISER H.W., DONKER J.D., 1968. Forage evaluation II - Productive response of first-calf Holstein Heifers fed early-cut or late-cut Alfalfa hay as the only source of energy throughout a total lactation. J. Dairy Sci., 51, 367-372.

MONTEIRO L.S., 1972. The control of appetite in lactating cows. Anim. Prod., 14, 263-281.

MURDOCK F.R., HODGSON A.S., 1969. Input-output relationships of cows fed two types of roughage and two levels of concentrate during complete lactations. J. Dairy Sci., 52, 1961-1972.

OLDENBROEK J.K., 1984. Holstein friesians, Dutch friesians and Dutch red and whites on two complete diets with a different amount of roughage : performance in first lactation. Livest. Prod. Sci., 11, 401-415. 